\title{
The NIRSPEC Brown Dwarf Spectroscopic Survey
}

\author{
Ian S. McLean, M. R. McGovern, Lisa Prato, Adam J. Burgasser \\ UCLA, Dept. Physics \& Astronomy, Los Angeles, CA 90095 \\ J. Davy Kirkpatrick \\ Infrared Processing and Analysis Center, California Institute of \\ Technology, Pasadena, CA 91125
}

\begin{abstract}
This paper describes the status of a new $0.9-2.4 \mu \mathrm{m}$ spectroscopic survey of low-mass stars and brown dwarfs. Flux-calibrated spectra of 30 objects from M6 to T8 have been obtained with a uniform spectral resolution of $\mathrm{R}=2,000$. For the $\mathrm{J}$-band alone, 60 objects have been observed. In addition, we have obtained the first sequence of spectra at $R=20,000(15 \mathrm{~km} / \mathrm{s})$ showing the detailed behavior of the J-band potassium doublets over the same range in spectral type. A few sources have been monitored for radial velocity variations, but none have been detected at the $\pm 1-2 \mathrm{~km} / \mathrm{s}$ level. Preliminary infrared spectral classification indices have been derived and several young brown dwarfs show clear spectral signatures of lower gravity. Our survey will be available on-line in the future.
\end{abstract}

\section{Introduction}

The NIRSPEC Brown Dwarf Spectroscopic Survey (BDSS) was established as one of the Key Projects for the Near-Infrared Spectrometer (NIRSPEC) on the Keck II 10-m telescope (McLean et al. 1998, 2000a). Two spectral modes are available: a low-resolution $(\mathrm{R} \sim 2,000)$ long-slit $\left(42^{\prime \prime}\right)$ mode and a high-resolution ( R 20,000) cross-dispersed echelle mode. The BDSS, a joint project with members of the 2MASS team, began shortly after NIRSPEC was delivered and commissioned in April 1999, and the initial phase of the survey is now nearing completion. Our primary goal was to obtain a uniform sequence of nearinfrared (NIR) spectra, with high signal-to-noise and resolution significantly better than $R \leq 800$ in order to extend and develop infrared (IR) classification of brown dwarfs, generate comparisons with model atmospheres, and enable more detailed physical studies of individual sources.

Because the J-band $(1.14-1.35 \mu \mathrm{m})$ contains both atomic and molecular features suitable for classification, we began by obtaining low-resolution $(R \sim 2,000)$ spectra of at least two examples of every spectral class from M6 to T8 (where possible). This basic set was then extended to include flux-calibrated spectra with continuous coverage from 0.9-2.4 $\mu \mathrm{m}$ for all even-numbered spectral classes in the same spectral type range. A subset of the brighter objects was observed 


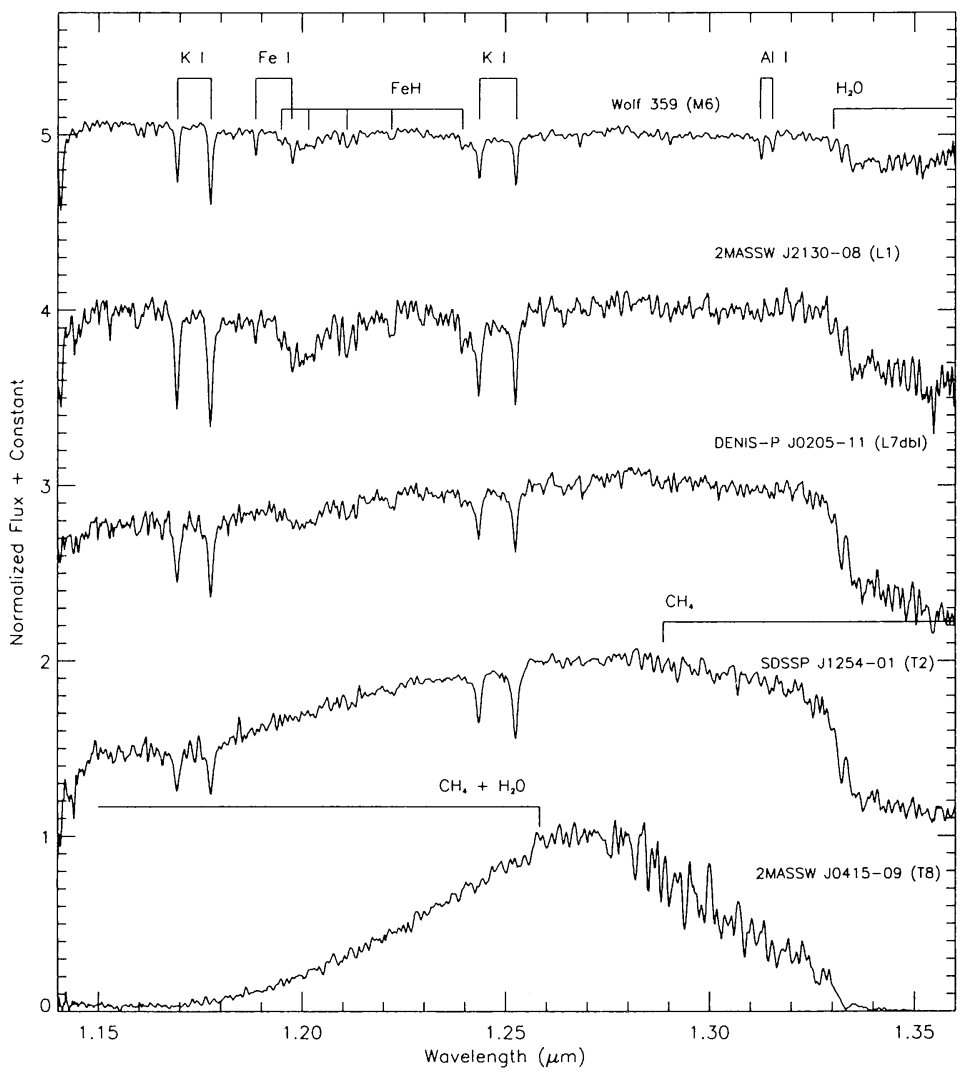

Figure 1. Five NIRSPEC J-band spectra from our survey (M6 to T8) illustrate variations of the prominent spectral features.

at resolutions over $\mathrm{R} \sim 20,000(15 \mathrm{~km} / \mathrm{s})$ to provide the first high-resolution sequence showing the development of pressure-broadening in the J-band neutral potassium lines. Several sources were also observed on multiple occasions to look for radial velocity variations. In addition, young, double or otherwise peculiar systems of brown dwarfs have been studied. Currently, the BDSS consists of 62 objects observed in $\mathrm{J}, 31$ in $\mathrm{H}$ and 34 in K. Early results were reported in McLean et al. (2000b) and McLean et al. (2001).

\section{Results and Discussion}

The source sample was selected from the 2MASS data base (Kirkpatrick et al. 2000; Burgasser et al. 2002), augmented with discoveries from DENIS and SDSS. Typically, 20 minute integrations (for $\mathrm{J} \sim 15$ ) in six grating settings provided complete spectral coverage from $0.9-2.4 \mu \mathrm{m}$. For all low-resolution observations a 2-pixel $\left(0.4^{\prime \prime}\right)$ slit was used, yielding $R \sim 2,000$; these spectra have been fluxcalibrated using 2MASS photometry (McLean et al. 2001). NIRSPEC data 

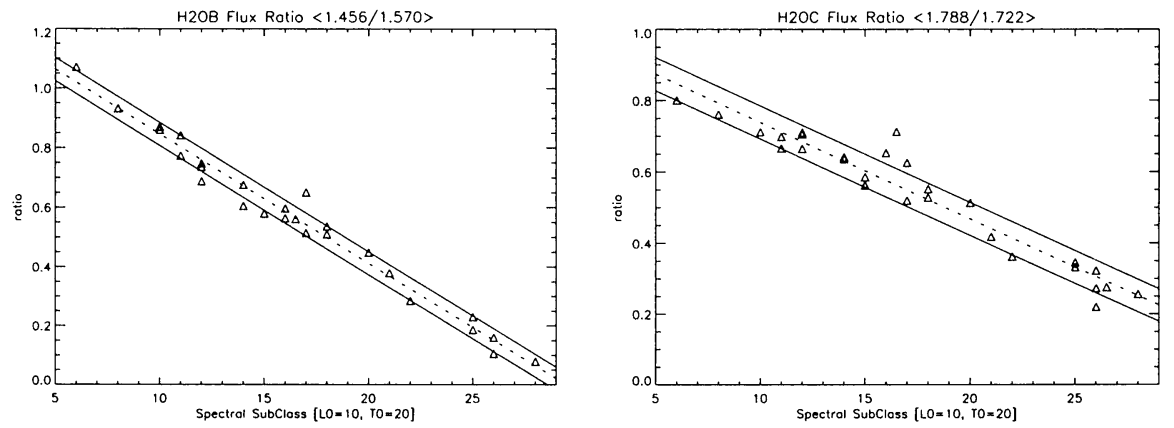

Figure 2. These $\mathrm{H}_{2} \mathrm{O}$ ratios exhibit a good correlation with previously derived spectral types, yielding an average deviation of 0.5 spectral class.

reduction is also described in that paper. Figure 1 shows a sub-sample of objects from our J-band library to illustrate the resolution and quality of the data.

The low-resolution spectra have been used to define carefully a series of flux ratios for spectral classification. Four ratios are associated with the $\mathrm{H}_{2} \mathrm{O}$ steam bands, two with methane $\left(\mathrm{CH}_{4}\right)$, one with $\mathrm{CO}$ and one with $\mathrm{FeH}$. In all cases, the ratios are defined in terms of a median flux value over a $0.02 \mu \mathrm{m}$ window centered on the given wavelength. The four $\mathrm{H}_{2} \mathrm{O}$ bands are designated by the letters $\mathrm{A}, \mathrm{B}, \mathrm{C}$ and $\mathrm{D}$ and the associated flux ratios correspond to the wavelengths (in $\mu \mathrm{m}$ ) 1.343/1.313, 1.456/1.570, 1.788/1.722 and 1.964/2.075. For $\mathrm{CH}_{4}$, the wavelengths used for the flux ratios are 1.730/1.590 and 2.200/2.100. A computer program was used to optimize these ratios.

Figure 2 shows two of the most useful indices plotted against L spectral types from Kirkpatrick et al. (2000) and T spectral types from Burgasser et al. (2002). In general, the fits are smooth and linear, with deviations of $<0.5$ spectral classes. We are currently completing observations and analysis of dwarf spectral standards which will be used to define a pure NIR classification scheme.

Figure 3 shows a subset of the Survey (subtypes M9 to T5) for which high resolution spectra with an average resolving power of $20,000(15 \mathrm{~km} / \mathrm{s})$ have been obtained. In echelle mode, typical observation times are 40 minutes with a slit width of $0.43^{\prime \prime}$. Only one of the 11 orders spanning the J-band is shown. This order (65) contains the potassium (K I) doublet near $1.177 \mu \mathrm{m}$. The 8 spectra in the sequence have been shifted to a common rest wavelength and displaced vertically for clarity. The signal-to-noise ratio is high; even for the T5 object, most features are real. Pressure broadened line widths exceed $500 \mathrm{~km} / \mathrm{s}$. Rotational velocities appear to be $20-30 \mathrm{~km} / \mathrm{s}$ wide in the $\mathrm{L}$ dwarfs. Multiple observations of several objects reveal no radial velocity variations at the 1-2 $\mathrm{km} / \mathrm{s}$ level.

In summary, a uniform sequence of low and high resolution near-IR spectra has been obtained by the NIRSPEC BDSS for spectral types M6 to T8. These provide a rich source for modeling and understanding the atmospheres of brown dwarfs. The optimal flux ratios derived from the spectra establish a NIR spectral classification scheme; high resolution spectra help constrain model atmospheres. 


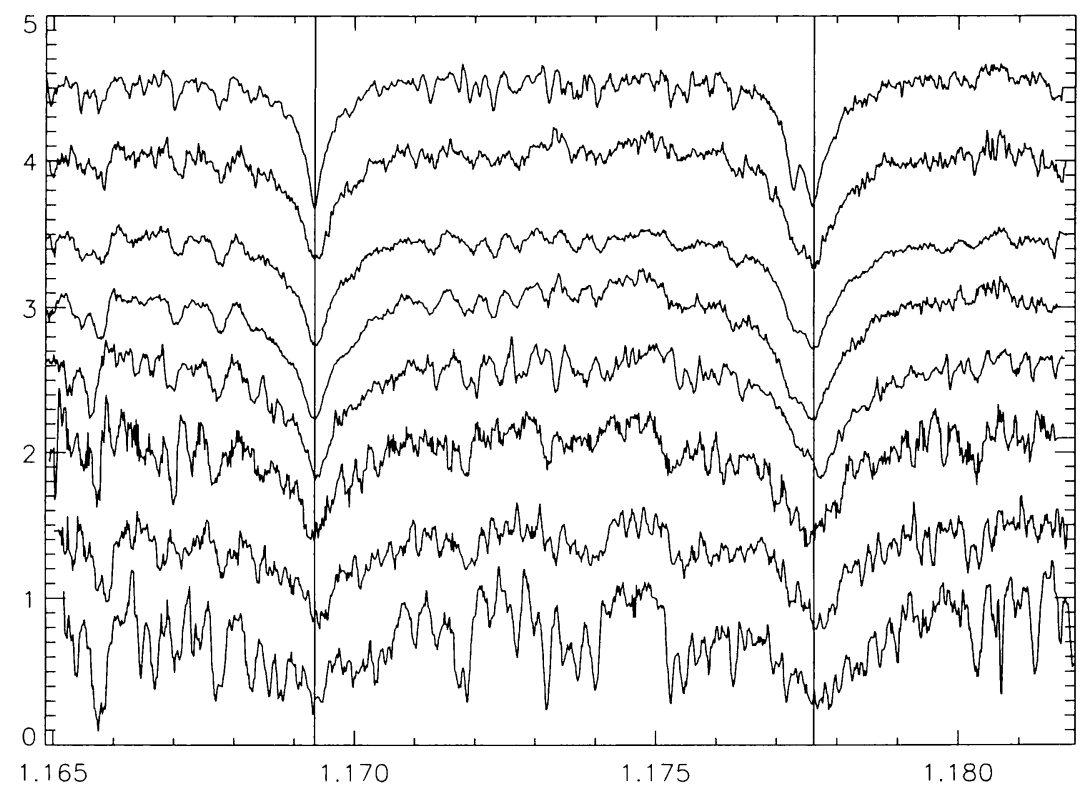

Figure 3. R 20,000 NIRSPEC spectra showing pressure broadening in a K I doublet. Spectra are normalized to 1.0 and displaced vertically for clarity.

With some exceptions, there is a good correlation between spectral types based on far red optical features and those derived from IR features. Comparison between the NIR and optical subtypes may reveal interesting properties, such as the presence of unseen companions, variable cloud condensation effects, or low gravity features indicative of youth. A more detailed paper is currently in preparation (McLean et al. 2003) and the results of the survey will also be made available in digital form in the near future.

Acknowledgements: This work was supported by the California Association for Research in Astronomy which operates the Keck Observatory on behalf of UC, Caltech, UH and NASA. AJB acknowledges support of a Hubble Fellowship.

\section{References}

Burgasser, A.J., et al. 2002, ApJ, 564, 421

Kirkpatrick, J.D., et al. 2000, AJ, 120, 447

McLean, I.S., et al. 1998, Proc. SPIE, 3354, 566

McLean, I.S., et al. 2000a, Proc. SPIE, 4008, 1048

McLean, I.S., et al. 2000b, ApJ, 533, L45

McLean, I.S., et al. 2001, ApJ, 561, L115 